\title{
Characterization of railway traffic and its effects on a short span bridge by using a hybrid fibre optic / electrical measurement system
}

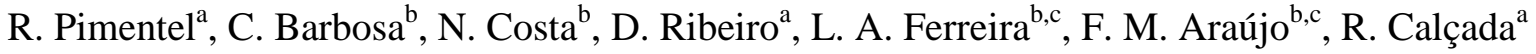 \\ ${ }^{a}$ Dep. Eng. Civil da Fac. de Eng. da Univ. do Porto, Rua Dr. Roberto Frias, s/n, 4200-465 Porto, Portugal \\ ${ }^{\mathrm{b}}$ FiberSensing, R. Vasconcelos Costa, 277, 4470-640 Maia, Portugal \\ ${ }^{c}$ INESC Porto, Campus da FEUP, R. Dr. Roberto Frias, 378, 4200-465 Porto, Portugal
}

Corresponding author: C. Barbosa, cristina.barbosa@ fibersensing.com

\begin{abstract}
The characterization of traffic effects on a short span railway bridge in Northern Portugal with a new hybrid platform that allows the simultaneous assessment of signals generated by a sensing network composed of both electrical and fibre Bragg grating based sensors was demonstrated. A Bridge Weight-in-Motion algorithm was also developed, which allows on-motion determination of train speed and weight distribution with only three fibre Bragg grating sensors.
\end{abstract}

Keywords: fibre Bragg gratings, sensors, measurement units, railway, bridges, Bridge Weight-in-Motion.

\section{INTRODUCTION}

The knowledge of the dynamic effects on railway bridges is of major importance for the following reasons: the vibrations induced by the passage of the trains over the bridge originate, in general, displacements or internal stresses in structures greater than those produced when the loading is statically applied; excessive vibrations of the structure may lead to a magnification of fatigue phenomena; the deformations and accelerations of the bridge should be controlled and kept within certain limit values, in order to ensure the stability of the track and of the contact wheel-rail at all times; the accelerations in the vehicles should be limited so that the passengers comfort can be guaranteed ${ }^{[1]}$. Simultaneously, the ability to determine train characteristics from its velocity to its weight distribution as a real time application is something that is gathering increasing interest, for it allows the responsible entity for the infrastructures to actually know applied loads and to have a better control of the operating companies. One of the used processes to determine traffic characteristics is the Bridge Weight-in-Motion (B-WIM) system ${ }^{[2]}$.

In the last few years, the examples of application of fibre Bragg grating sensors for structural health monitoring have become more and more frequent ${ }^{[3,4]}$. The explanation for this fact relies not only on the particularly appropriate characteristics associated to these devices for measuring the most relevant structural parameters, without revealing a number of disadvantages usually attributed to conventional sensors, but also on the growing range of available sensors based on this technology, as well as several types of measurement units with increasing capabilities. In particular, fibre Bragg sensors are most suited for measuring strain in rail track applications where EM/RF noise often compromises the performance of electrical strain gages ${ }^{[5,6]}$.

Nevertheless, for the complete dynamic characterization of a structure like a railway bridge, acceleration measurements are mandatory. These can be performed with standard electrical accelerometers, readily available with a diversity of characteristics. However, measurements gathered with fibre optic and electrical sensors must be synchronized, in order to provide useful data.

In this work, it is shown the applicability of a new measurement unit based on a hybrid platform concept, which allows the simultaneous assessment of signals generated by the fibre Bragg grating sensors used to measure strain in the rail and bridge elements, as well as the electrical accelerometers. These units were developed with a graphical interface that allows the user to configure optical and electrical sensors in a similar way, as well as acquire, operate and store both types of signals. The units are expansible through a built-in PXI sub-rack, being possible to increase the number of electrical and optical channels by inserting additional optical switching modules or PXI data acquisition cards.

In addition, on-motion determination of train compositions weight, as well as its distribution, with a simple dynamical measurement system based on three fibre Bragg grating sensors and an algorithm specifically developed for this purpose was demonstrated. 


\section{BRIDGE DESCRIPTION}

Canelas Bridge is situated on $\mathrm{Km}+282.944$ of the "Linha do Norte" of the Portuguese railway network. This piece of the national railway system has recently been modernized to allow faster train circulation - the "Alfa Pendular" train can reach up to $220 \mathrm{~km} / \mathrm{h}$. The Canelas Bridge is a Filler Beam Bridge with a concrete slab with embedded steel profiles. The bridge's cross section is composed of two symmetric and independent half decks, each supporting one of the railway tracks. There are six simply supported spans with approximately $12 \mathrm{~m}$ wide. On Figure 1 a picture of the bridge and its cross section are presented.

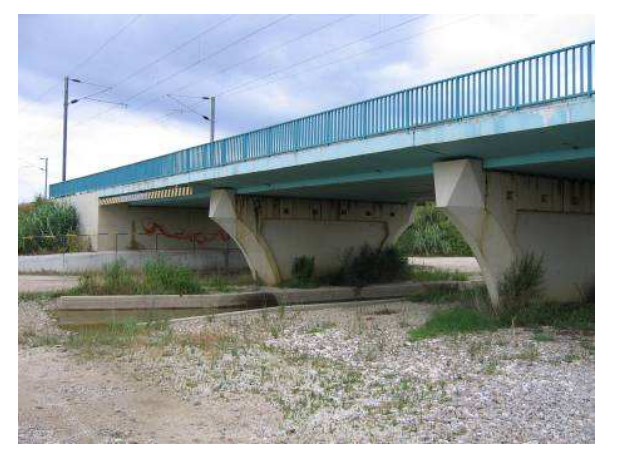

a)

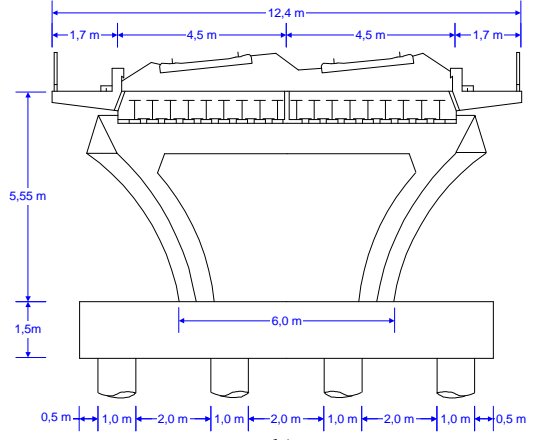

b)

Figure 1. Canelas Bridge: a) side view; b) cross section.

\section{MEASUREMENT SYSTEM}

The measurement unit used on the experimental campaigns is based on the BraggScope technology, which combines a high-power broadband optical source with proven thin-film optical filtering technology in an intelligent approach to perform dynamic measurement of the Bragg wavelength. By integrating proprietary add/drop WDM design, it allows the measurement of up to four sensors connected in series, operating within predefined wavelength bands. This, in combination with optical switching makes this measurement technology a cost effective solution for medium scale dynamic sensing networks.

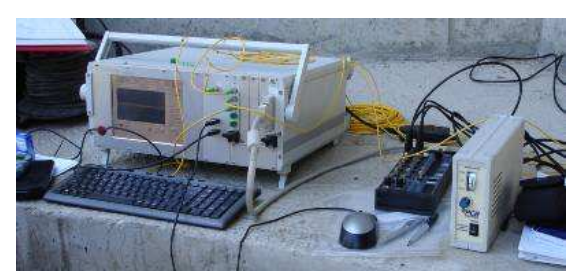

Figure 2. Hybrid measurement unit used in the experimental campaigns.

The BraggScope benchtop measurement units are expansible through a built-in PXI sub-rack, so it is possible to increase the number of electrical and optical switching modules or PXI standard data acquisition cards. This is the socalled hybrid concept, which allows the simultaneous assessment of signals generated by a sensing network composed by both electrical and fibre Bragg grating sensors (Figure 2). Through the unit touch-screen graphical interface, it is possible for the user to configure optical and electrical sensors in a similar way, as well as acquire, operate and store both types of signals.

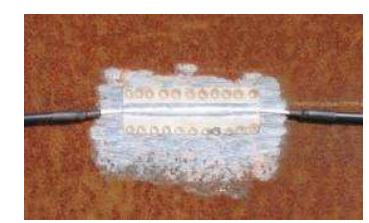

Figure 3. Fiber Bragg grating weldable strain sensor on the rail.

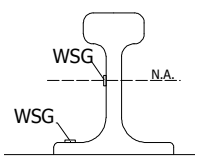

Figure 4. Sensor position on the rail.

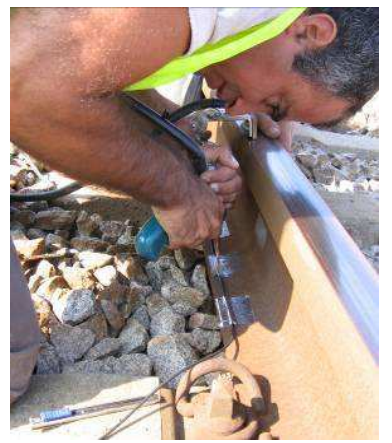

Figure 5. Sensor installation.

Strain and displacement sensors based on fibre Bragg gratings were used on the experimental campaigns. For strain measurements, a weldable fibre Bragg grating strain gauge was applied. This type of sensor is clearly preferable since it minimizes surface preparation requirements and precludes epoxy curing processes during installation. The spot welding process has no implications on the rail tracks for it consists on creating an electrical current between two close points. The weldable fibre Bragg grating strain gauge (Figure 3) is based on a capillary stainless steel tube containing the sensing element, which is laser welded to a thin stainless steel base that is used for direct spot welding to the metallic structure. The input/output fibres are protected with standard $900 \mu \mathrm{m}$ buffer. For permanent installations, this buffer is further protected by a $3 \mathrm{~mm}$ armoured cable, while the sensors can be covered with a metallic cap also spot weldable to the structure ${ }^{[7]}$. For the first testing campaign, strain was controlled under the bridge on the mid-span of two consecutive spans aligned with both rail. Strain was also monitored on the rail with two sensors, being one of them deployed along the neutral axis and the other one on the basis of the rail (Figure 4), mainly for separating and evaluating the effects of rail track deformation due to total deflection of the deck and to train wheels loading. 
On the second campaign, aimed to calibrate the B-WIM algorithm, two weldable fibre Bragg grating strain gages were spot-welded to the basis of the rail in two consecutive spans and another two were positioned under the bridge, on the mid-span, aligned with both rails. Vertical displacements were obtained using optical long gauges (Figure 6). The long gauges consist on a tensioned invar wire, which strain variations are measured with a fibre Bragg grating and integrated along its length for displacement determination.

The tension had to be adjusted so that the wire vibration modes did not

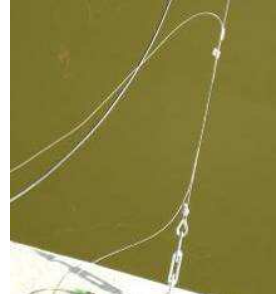

Figure 6. Long gauge.

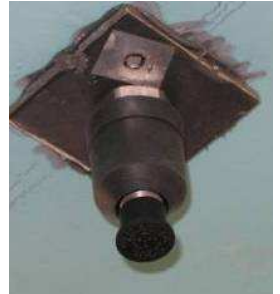

Figure 7. Accelerometer. coincide with the bridge resonances. Vertical displacements were controlled in two consecutive mid spans and on one bearing. Accelerations were measured with traditional piezoelectric accelerometers (Figure 7).

\section{EXPERIMENTAL CHARACTERIZATION OF RAILWAY TRAFFIC}

The characterization of railway traffic was performed through the implementation of a B-WIM algorithm. This algorithm allows the evaluation of the train's speed, acceleration, longitudinal position of each axle and its respective load. Firstly, axle position is detected using the acquisition of longitudinal deformation of one point of the rail track. The velocity and acceleration of the train can then be determined using deformation of the track in a different position at a known distance from the first. Secondly, loading per axle is calculated. This is possible using a third measurement of deformation, this time under the deck. The registered data is filtered using a low-pass digital filter to eliminate any dynamic effect on the response and used as an input for a Moses' Algorithm ${ }^{[2]}$.

On Figure 8 the register for the deformation on two different points on the rail (E5 and E7) is presented. The shown event represents the passage of the locomotive and of the first carriage on an intercity train travelling at $88 \mathrm{~km} / \mathrm{h}$. Figure 9 shows the deformation at the mid-span of one metallic beam of the deck aligned with one of the rails for the whole train passage (E4).

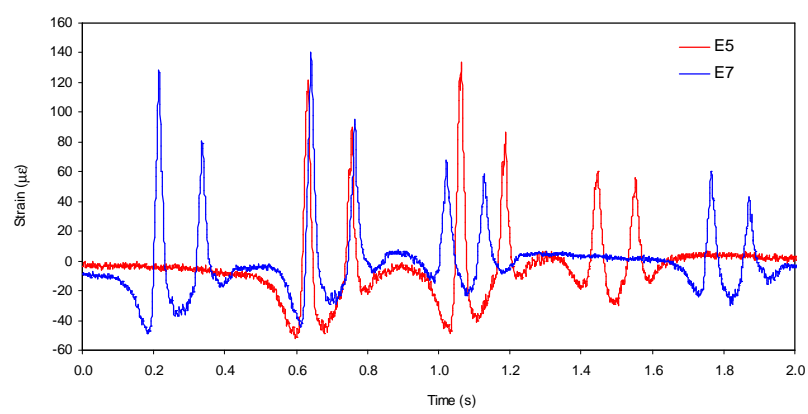

Figure 8. Deformation in two different points on the rail track.

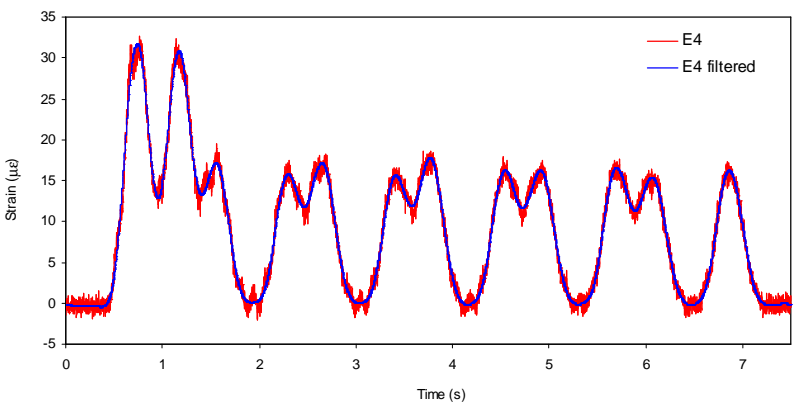

Figure 9. Deformation in one point of the deck.

The results obtained by the application of the algorithm are shown in Figure 10 and Figure 11. The first picture represents the geometry and loads per axle. The second one shows the experimental determination of the influence line of deformation of the strain gauge on the beam. This determination was possible for a well characterized train (geometry and loading) passing at a low speed on the bridge and using the Matrix Type Method ${ }^{[8]}$.

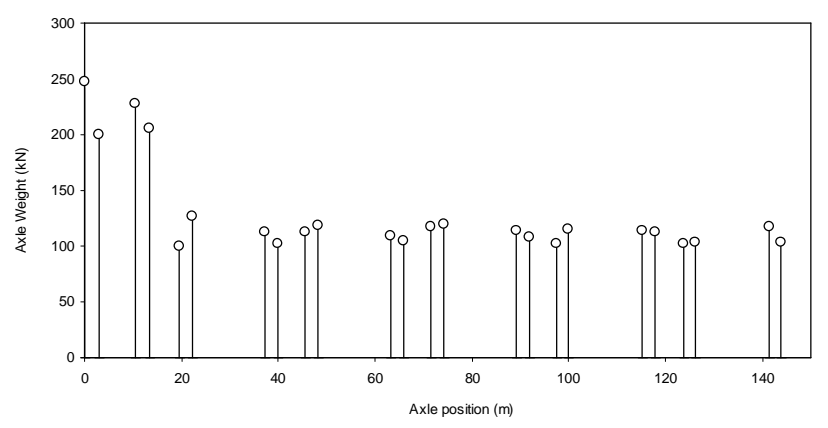

Figure 10. Position and load of each axle in an intercity train.

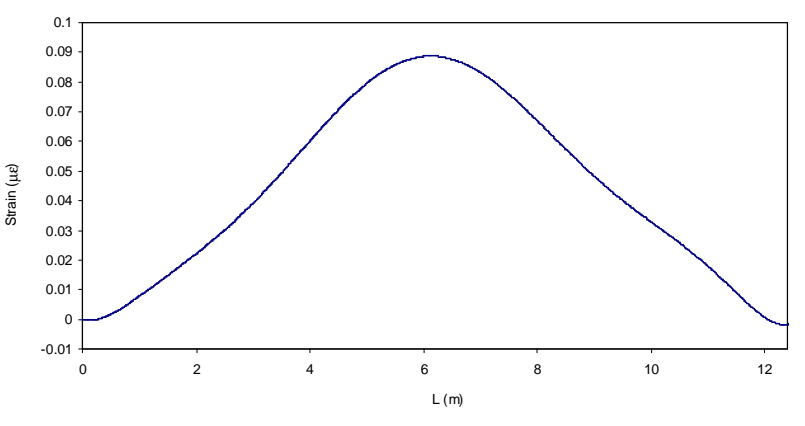

Figure 11. Influence line of deformation of the deck. 


\section{EXPERIMENTAL EVALUATION OF TRAFFIC EFFECTS}

The importance of the dynamic effects induced by traffic loads on bridges is usually measured by the so-called dynamic amplification factor, which can be defined as the ratio between the maximum dynamic response of the bridge and the corresponding maximum static response. The maximum dynamic response is directly obtained by taking the maximum value of the measured data. On the other hand, the maximum static response can be calculated by applying a digital low-pass filtering to the measured time series, so as to eliminate the corresponding dynamic components, as referred in the previous section. Taking notice on Figure 12, we can verify that the dynamic amplification due to the passage of an intercity train at $88 \mathrm{~km} / \mathrm{h}$ is low. For ballasted bridges it is necessary that the maximum acceleration of the deck does not exceed $0.35 \mathrm{~g}$ to assure the ballast stability. The data in Figure 12 shows that the maximum acceleration for frequencies below $30 \mathrm{~Hz}$ is always lower than the referred limit. Nevertheless, the evaluation of the fatigue behaviour of the structure is an important matter. In Figure 13 the strain spectrum on the metallic beam for the passage of the intercity train is presented. The spectrum was obtained using the Rainflow counting method ${ }^{[9]}$. Adopting an appropriate $\mathrm{S}-\mathrm{N}$ fatigue resistance curve we can determine the individual damage associated to the train passage. For the passage of more than one train, the damage can be calculated using the Palmgren Miner rule of linear accumulation of damage ${ }^{[9]}$. With this information, life expectancy and total damage of the structure can be estimated.

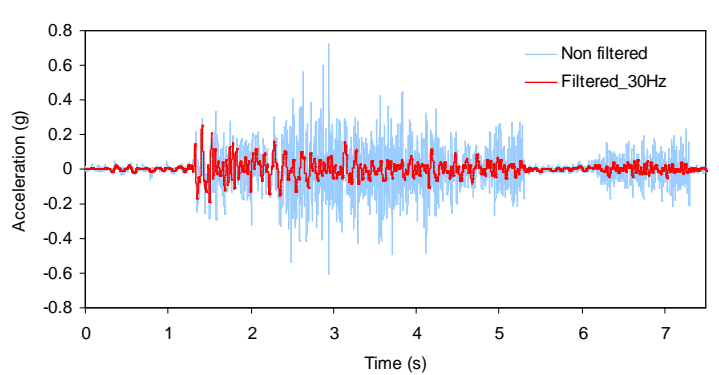

Figure 12. Vertical acceleration of the deck for the intercity train.

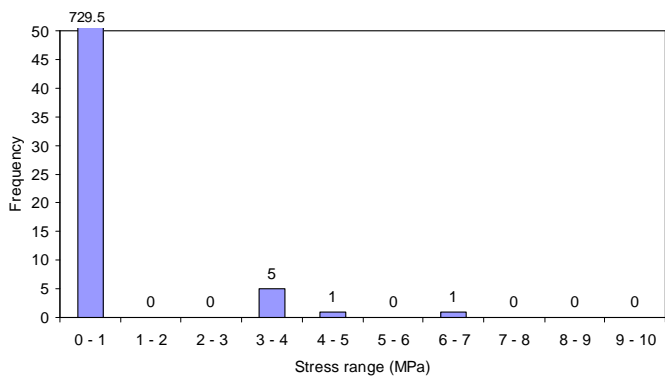

Figure 13. Spectrum of strain on a deck beam for the intercity train.

\section{CONCLUSIONS}

In this communication, the characterization of traffic effects on a short span railway bridge in Northern Portugal by using a new hybrid platform that allows the synchronous assessment of signals generated by an electrical/fibre Bragg grating sensing network was described. With data collected during the experimental campaigns, a new Bridge Weight-inMotion algorithm was developed. The obtained results allowed us to design a competitive complete system for onmotion determination of train speed and weight distribution based in only three fibre Bragg grating sensors and a single optical channel measurement unit.

\section{ACKNOWLEDGMENTS}

The authors acknowledge the support and collaboration provided by Eng. Ana Isabel Silva from REFER.

\section{REFERENCES}

1. R. Delgado, R. Calçada, I. Faria. "Bridge-vehicles dynamic interaction: numerical modelling and practical applications", Bridges for HighSpeed Railways, Porto, Portugal, 3-4 June, 2004.

2. F. Moses, "Weigh-in-Motion System using instrumented Bridges", ASCE Transportation Engineering Journal, 105, TE3, 233-249, 1979.

3. K. Kincade, "Fibre sensors lay groundwork for structural health monitoring", Laser Focus World 42 (2), 63-67 (2005).

4. Sensing issues in civil structural health monitoring, F. Ansari, ed. (Springer, Dordrecht, 2005).

5. P. Boffi, R. Bratovich, F. Persia, A. Barberis, M. Martinelli, A. Basso, G. Bucca, A. Nicchio, M. Bocciolone, "Fibre sensor for collector strain monitoring in the pantograph-catenary interaction", 18th Int. Conference on Optical Fibre Sensors, Cancun, 23-27 October 2006.

6. Chung-Yue Wang, Hao-Lin Wang, Ming-Hung Chen, "Structural health monitoring activities of applying optical fibre sensors in Taiwan", 18th Int. Conference on Optical Fibre Sensors, Cancun, 23-27 October 2006.

7. C. Barbosa, N. Costa, L. A. Ferreira, F. M. Araújo, H. Varum, A. Costa, "Monitoring the new circular pedestrian steel bridge over the São Roque and Botirões channels with weldable fibre-Bragg grating sensors", 18th Int. Conference on Optical Fibre Sensors, Cancun, 23-27 October 2006

8. M. Quilligan, "Bridge weigh-in-motion, development and testing of a 2-dimensional multi-vehicle algorithm", Licentiate thesis, Royal Institute of Technology, Stockholm, 2003.

9. D. Ribeiro, "Comportamento dinâmico de pontes sob acção de tráfego ferroviário a alta velocidade", M.Sc. Thesis, FEUP, Porto (2004). 